\title{
RIGIDEZ ARTERIAL: APLICAÇÕES CLÍNICAS DOS CONCEITOS E MÉTODOS DE AVALIAÇÃO
}

\author{
ARTERIAL RIGIDITY: CLINICAL APPLICATIONS OF ASSESSMENT CONCEPTS \\ AND METHODS
}

Antônio Carlos de Souza Spinelli', Vanildo Guimarães²

\section{RESUMO}

A caracterização de rigidez arterial é realizada a partir da análise de alterações nas propriedades físicas da parede arterial, entre elas, distensibilidade, complacência e elasticidade. O aumento da rigidez arterial leva a arteriosclerose que está associada ao envelhecimento e a presença dos fatores de risco cardiovasculares tradicionais determinando alterações no padrão de fluxo nas artérias elevando o risco de progressão da aterosclerose. A aterosclerose promove uma solução de continuidade no endotélio que ao final leva a degeneração elástica na parede arterial e o enrijecimento da parede vascular. Essa é a condição determinante para a perda do mecanismo de adaptação ao volume ejetado na sístole ventricular e à onda de retorno na fase de diástole, fato observado principalmente nas grandes artérias elásticas como a aorta e as carótidas. Os principais fatores envolvidos neste processo são a hiperatividade simpática, o estado inflamatório crônico do vaso e a redução da biodisponibilidade do oxido nítrico. Vários métodos estão disponíveis para avaliar a pressão central e parâmetros de rigidez arterial. O método direto seria o de maior precisão, no entanto, é um método invasivo, e claramente inadequado para uso na avaliação clínica de rotina. Atualmente dispomos de métodos indiretos com adequada aplicação na determinação de índices de enrijecimento das grandes artérias. Um desses métodos permite a avaliação da pressão arterial central (PAc), velocidade da onda de pulso (VOP), e medidas do índice de aumentação (Alx) que são marcadores bem estabelecidos da hemodinâmica central e da rigidez arterial, e nos oferece uma visão importante da vitalidade arterial. Entre esses índices, a VOP se apresenta como um marcador de dano vascular, com elevada importância na determinação do risco cardiovascular global dos pacientes, assim como o Alx, outro parâmetro de envelhecimento vascular, ambos preditores de mortalidade por todas as causas e por causas cardiovasculares. As diferentes classes de anti-hipertensivos têm efeitos diversos sobre a hemodinâmica central e a correta interpretação dos achados obtidos nos exames de avaliação dos parâmetros centrais pode nortear mais adequadamente a estratégia do tratamento da hipertensão arterial.

Descritores: Rigidez Arterial; Arteriosclerose; Velocidade da Onda de Pulso; Anti-Hipertensivos.

\section{ABSTRACT}

The characterization of arterial stiffness is performed by analyzing changes in the physical properties of the arterial wall, including distensibility, complacency and elasticity. The increase in arterial stiffness leads to arteriosclerosis that is associated with aging and the presence of traditional cardiovascular risk factors and causes changes in the flow pattern in the arteries, increasing the risk of atherosclerosis progression. Atherosclerosis causes an endothelium injury that ultimately leads to elastic degeneration in the arterial wall and the stiffening of the vascular wall, a determining condition for the loss of the ability to adapt to the volume ejected in the ventricular systole and the return wave in the diastole phase, a fact observed mainly in large elastic arteries such as the aorta and carotids. The main factors involved in this process are sympathetic hyperactivity, the chronic inflammatory state of the vessel and the reduction of nitric oxide bioavailability. Several methods are available to assess central pressure and arterial stiffness parameters. The direct method is the most accurate. However, it is an invasive method, and clearly unsuitable for use in routine clinical evaluation. Currently we have indirect methods which are perfectly applicable for the determination of stiffening indexes of the great arteries. One of these methods allows the assessment of central arterial pressure (PAC), pulse wave velocity $(P W V)$, and measures of the augmentation index (Alx), which are well-established markers of central hemodynamics and arterial stiffness, and provides an important assessment of arterial vitality. Among these indices, PWV consists of a marker of vascular damage, highly important in determining the overall cardiovascular risk of patients, as well as Alx, another parameter for assessing vascular aging, both of them functioning as predictors of risk of mortality from all causes and from cardiovascular causes. The

1. Universidade Federal do Rio Grande do Norte- Natal (RN), Brasil Cardiocentro- Natal (RN), Brasil.

2. Hospital Getúlio Vargas - Recife (PE), Brasil. Diagnóstico Cardíaco - Recife (PE), Brasil.

Correspondência: Antônio Carlos de Souza Spinelli. Rua Campos Sales, 742 Tirol. CEP 5902O-300. Natal, RN, Brasil. spinelli@cardiol.br Vanildo Guimarães. Rua do Futuro, 141, Graças, CEP: 52050-010, Recife, PE, Brasil. vanildo@cardiol.br

http://dx.doi.org/10.47870/1519-7522/202027017-12 
different classes of antihypertensive drugs have different effects on central hemodynamics and the correct interpretation of the findings obtained in the assessment tests of the Central Parameters can more adequately guide the strategy for the treatment of arterial hypertension.

Keywords: Arterial Stiffness; Arteriosclerosis; Pulse Wave Analysis; Antihypertensive Agents.

\section{CONSIDERAÇÕES INICIAIS}

A análise das alterações observadas nas propriedades físicas da parede arterial, como a distensibilidade, complacência e elasticidade, nos permite caracterizar a rigidez arterial. Essas alterações resultam em modificações na fisiologia arterial, uma vez que afetam a forma como as artérias se adaptam à pressão e ao fluxo sanguíneo a cada batimento cardíaco. ${ }^{1}$

O aumento da rigidez arterial leva a arteriosclerose, uma doença da túnica média arterial que está associada ao enveIhecimento ${ }^{2}$ e que apresenta uma velocidade de instalação acelerada na presença dos fatores de risco cardiovascular tradicionais como, hipertensão arterial, diabetes mellitus tipo 2, obesidade e dislipidemia. ${ }^{3}$

As alterações no padrão de fluxo nas artérias elásticas causadas pela rigidez arterial, principalmente, o aumento do pico de pressão sistólica e a redução do fluxo diastólico que são responsáveis pelo estresse hemodinâmico, eleva o risco da progressão da aterosclerose e desenvolvimento de obstruções arteriais. ${ }^{1}$

A avaliação da pressão arterial central (PAc), velocidade da onda de pulso (VOP), e medidas do índice de aumentação (AIx) (Figura 1) são marcadores bem estabelecidos da hemodinâmica central e da rigidez arterial e nos oferece uma visão importante da vitalidade arterial. ${ }^{4-7}$ Esses parâmetros podem adicionar valor preditivo à estimativa de risco cardiovascular já demonstrada pelas clássicas medições periféricas da pressão arterial. ${ }^{8-10}$

Neste artigo de atualização revisamos as recentes publicações sobre rigidez arterial, avaliação dos parâmetros centrais e a aplicação destes conceitos na prática clínica corrente, buscando demonstrar a contribuição dos métodos na propedêutica cardiovascular.

\section{FISIOPATOLOGIA DA RIGIDEZ ARTERIAL}

A arteriosclerose leva ao endurecimento das artérias por um processo degenerativo da túnica média das artérias elásticas que se desenvolve com o envelhecimento e, como já citado, é potencializado por fatores de risco cardiovascular, ${ }^{3}$ O principal fator para todo esse acontecimento é um estado

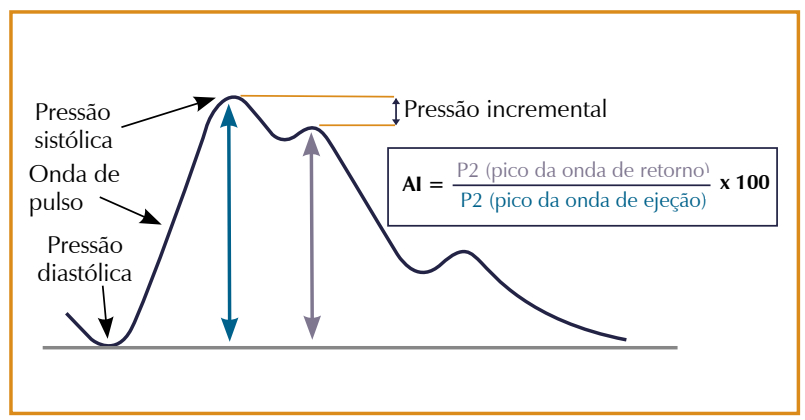

Figura 1. VOP e Alx. inflamatório crônico do vaso, em resposta ao estresse oxidativo mitocondrial da célula muscular. ${ }^{11}$ A arteriosclerose impulsiona o espessamento da parede das artérias, redução da complacência e aumento da rigidez, resultando na instalação do quadro de aterosclerose, desordem que leva a formação de placas na camada íntima vascular. As placas são depósitos de lipídeos, células inflamatórias, tecido conjuntivo fibroso, células musculares lisas e cálcio, que progressivamente atuam na redução do lúmen arterial. ${ }^{2}$

A aterosclerose, ao produzir uma solução de continuidade no endotélio facilitando o depósito de lipídeos e expressão de citocinas inflamatórias, vai incrementar uma degeneração elástica na parede arterial, uma vez que potencializa o desarranjo estrutural da parede do vaso. Neste cenário fisiopatológico ocorrem o enrijecimento da parede vascular e perda do mecanismo passivo, provocando uma maior dificuldade de adaptação hemodinâmica ao volume ejetado na sístole ventricular e à onda de retorno na fase de diástole, fato observado principalmente nas grandes artérias elásticas como a aorta e as carótidas. ${ }^{12}$

A redução da biodisponibilidade do óxido nítrico, observada no envelhecimento e na aterosclerose, também se apresenta como outro fator importante que provoca o aumento do tônus vascular e redução do lúmen arterial. ${ }^{13}$ A ação vasodilatadora do óxido nítrico endotelial é um dos principais elementos que influenciam ativamente a vasodilatação arterial. A inibição de sua produção ou aceleração da sua inativação, que observamos em situações como o avanço da idade e a hipertensão arterial, levam ao remodelamento arterial e como resultado desses processos ocorre a uma redução na contratilidade vascular decorrente da degradação da elastina e deposição de colágeno. ${ }^{14,15}$

A aterosclerose representa o principal mecanismo na gênese da maioria das patologias isquêmicas, como infarto do miocárdio e acidente vascular encefálico isquêmico. ${ }^{3}$

\section{MANEIRAS DE MEDIR A PRESSÃO CENTRAL E OS PARÂMETROS DE RIGIDEZ ARTERIAL}

Vários métodos estão disponíveis para avaliar a pressão central e parâmetros de rigidez arterial. O método mais direto e preciso para medida da pressão central envolve o registro da pressão arterial na aorta ascendente através da colocação de um cateter intra-arterial que mensura a pressão nessa região. No entanto, é um método invasivo, tecnicamente exigente e claramente inadequado para uso na avaliação de rotina de grandes populações. Vários métodos não invasivos foram desenvolvidos, nos quais as formas de onda de pressão são registradas em locais distais à aorta, como as artérias carótida, braquial ou radial. Cada uma dessas abordagens tem seus próprios pontos fortes e limitações.

A pressão da artéria carótida é frequentemente usada 
como substituto da pressão aórtica, devido à proximidade desses locais arteriais. As formas de onda da pressão carotídea são registradas por tonometria de aplanação e, em seguida, dimensionadas para às pressões braquial média e diastólica, sob o princípio de que, diferentemente da pressão sistólica, pressão arterial média e pressão diastólica, não variam acentuadamente em toda a árvore arterial ${ }^{16} \mathrm{e}$, portanto, são adequadas para calibrar formas de onda de pressão registradas de outros sítios arteriais. A tonemetria é considerada padrão ouro entre os métodos não invasivos, contudo é onerosa e bastante dependente do operador, tornando-a inviável para a triagem rotineira da pressão central em um ambiente não especializado. ${ }^{17-19}$

A análise de ondas de pulso é um método alternativo, em que as formas de ondas de pressão são registradas a partir de artérias periféricas e a pressão aórtica central correspondente derivada é obtida através de algoritmos específicos e métodos matemáticos de calibração ou estimativa. Esses dispositivos oferecem vantagens potenciais por não serem tão dependentes do operador e são mais adequados para uso no ambiente de cuidados primários. ${ }^{20-22}$ Estudos recentes têm demonstrado que estes dispositivos automatizados com manguito servo-controlados, calibrados de forma não invasiva pelos valores da pressão arterial braquial média e diastólica, parecem oferecer uma abordagem mais promissora e podem fornecer uma estimativa não-invasiva precisa dos parâmetros centrais, podendo ser considerado um instrumento promissor na prática clínica. ${ }^{23,24}$

\section{RIGIDEZ ARTERIAL E SUAS IMPLICAÇÕES CLÍNICAS}

Inúmeras evidências têm demonstrado o efeito benéfico da diminuição da pressão arterial (PA) em reduzir o risco de doenças cardiovasculares (DCV) em hipertensos, no entanto, boa parte da redução do risco não pode ser explicada apenas pela redução da PA periférica, medida na maioria dos estudos na artéria braquial, onde não é representativo de toda a complexidade do processo hipertensivo. A partir daí surge o questionamento do efeito do tratamento anti-hipertensivo, além da redução da PA per se, atribuindo-se eventuais efeitos da medicação sobre propriedades vasculares independentes do efeito pressórico. As alterações da mecânica vascular de micro e macrocirculação não podem ser totalmente observadas pelas medidas de PA periférica. Assim, a avaliação da PA em outros segmentos da árvore arterial proporcionaria melhor visualização do benefício do tratamento sobre as alterações vasculares. ${ }^{25}$

Diversos métodos vêm sendo utilizados para determinação de índices de enrijecimento das grandes artérias. Um desses métodos tem trazido à luz conhecimentos clínicos de implicações diagnóstica, terapêutica e prognóstica na hipertensão arterial: a medida da velocidade de onda de pulso (VOP) e do índice de aumentação (Alx), que expressam à relação entre enrijecimento, elasticidade e complacência.

Na hipertensão arterial (HA), as artérias representam um dos órgãos alvo que podem ser lesados pela pressão arterial elevada; ${ }^{26}$ no entanto, o enrijecimento arterial também é considerado um fator causal que leva à hipertensão, pois precede e prediz a incidência desta última. ${ }^{27} \mathrm{O}$ marcador mais robusto e bem estudado da rigidez aórtica, a VOP carotídeo-femoral, demonstrou um valor preditivo incremental para eventos cardiovasculares futuros e mortalidade por todas as causas além dos escores clássicos de risco e da PA, que é o principal determinante modificável da rigidez aórtica. ${ }^{28,29}$ Além disso, existem algumas evidências sugerindo que uma VOP elevada está associada à uma resposta pobre dos medicamentos anti-hipertensivos sobre a $\mathrm{PA}^{30}$ e que a regressão da VOP está associada a uma melhor sobrevida. ${ }^{31,32}$

Estudos têm sugerido que a avaliação da pressão central pode trazer novas perspectivas e dados adicionais para a estratificação cardiovascular no manejo de pacientes com HA e DCV. Em um desses estudos, o Reason, os autores mostraram que a redução seletiva da PAc por tratamento anti-hipertensivo de longo prazo determina alterações estruturais da microcirculação que resulta alterações das ondas de reflexão e está associada a interações complexas entre pequenas e grandes artérias que, predominantemente, afetam os parâmetros hemodinâmicos centrais. ${ }^{33,34} \mathrm{O}$ desempenho hemodinâmico de grandes artérias deteriora-se com o envelhecimento como consequência de modificações estruturais na parede dos vasos e deterioração da função endotelial. ${ }^{35-42}$ Essas duas mudanças estruturais e funcionais são aceleradas em pacientes com $\mathrm{HA}^{42}$ e podem ser passíveis de modificação favorável por terapia medicamentosa. ${ }^{43}$

No estudo CAFE, que é um subestudo do ensaio clínico ASCOT, evidenciou que reduzir a PAc traz maior diminuição de risco cardiovascular, em comparação com a redução da PA periférica medida na artéria braquial. ${ }^{44}$ Dessa forma, a análise da PAc poderia proporcionar a avaliação de efeito diferencial dos medicamentos anti-hipertensivos sobre a árvore arterial, uma vez que a ação deles é diferente nas propriedades estruturais e funcionais vasculares, e na hemodinâmica central. ${ }^{43,44}$

A medida da VOP é aceita como padrão-ouro para avaliar a rigidez arterial. A PAc e o Alx sofrem maior influência de condições fisiopatológicas, medicamentos, frequência cardíaca e idade, o que torna a VOP mais fidedigna, podendo prognosticar mais eficientemente o aparecimento de complicações da hipertensão arterial, como o acidente vascular cerebral (AVC), o infarto do miocárdio (IM) e a doença renal crônica (DRC), uma vez que identifica mais precocemente alterações hemodinâmicas relacionadas a tais complicações. ${ }^{45,46}$

Estudo comparativo analisando diversos procedimentos de determinação da VOP na avaliação da rigidez arterial de indivíduos com diabetes mellitus (DM2) concluiu pela necessidade de maiores investigações para esclarecer sua utilidade nesse perfil de pacientes, contudo, reforçou a VOP como padrão-ouro também nessa população. ${ }^{47}$ Uma revisão sistemática que avaliou a relação da VOP com os diversos fatores de risco cardiovascular (RCV) mostrou que 52\% dos estudos encontraram uma associação positiva entre VOP aumentada e DM2. ${ }^{48}$

Além do efeito dominante do envelhecimento, outras condições fisiológicas e fisiopatológicas estão associadas com o aumento da rigidez arterial e mudança no padrão de comportamento da onda de pulso refletida. Entre elas, condições fisiológicas (baixo peso ao nascer, ciclo menstrual, menopausa), características genéticas (história familiar de HA, DM ou IM, e polimorfismos genéticos), fatores de risco cardiovascular (sedentarismo, obesidade, tabagismo, HA, dislipidemia, intolerância a glicose, síndrome metabólica, DM tipos 1 e 2), DCV e também doenças não cardiovasculares 
(DRC nos seus diversos estágios, artrite reumatoide, vasculite sistêmica, lúpus eritematoso sistêmico). ${ }^{49-51}$

Os critérios de rigidez arterial fornecem evidências diretas de danos em órgãos-alvo, sendo a VOP considerada um biomarcador de dano vascular, ${ }^{52}$ o que se reveste de importância na determinação do risco global cardiovascular do paciente, tendo em vista que os escores de risco clássicos, especialmente no extrato de risco intermediário, têm um desempenho ruim para predizer desfechos cardiovasculares. ${ }^{51,53,54}$ Uma metanálise de 17 estudos longitudinais que avaliaram a VOP e acompanharam 15.877 indivíduos por uma média de 7,7 anos, mostrou que rigidez arterial expressa pela medida da VOP é um forte preditor de eventos CV futuros e mortalidade por todas as causas. A capacidade preditiva da VOP na rigidez arterial é maior em indivíduos com maior risco basal de doença CV.

Aumento da VOP foi associada com maior volume de hiperintensidade da substância branca à RNM e à presença de infartos lacunares - $\mathrm{AVC}^{55}$ a aumento de mortalidade na $\mathrm{DRC}^{56}$ e aumento de mortalidade cardiovascular e por todas as causas, ${ }^{28}$ além de ter relação com o risco de pré-eclâmpsia e eclâmpsia na gestante. ${ }^{57}$

Diante do exposto, a medida da VOP está estabelecida como um adequado marcador de comprometimento vascular e como método preditor e prognóstico, permitindo, atualmente, a identificação precoce do comprometimento dos órgãos-alvo na HA. Estabelece-se, desse modo, a importância de se identificar indivíduos com risco elevado para tais alterações e, dessa forma, indicar uma intervenção terapêutica adequada. Uma VOP acima de $12 \mathrm{~m} / \mathrm{s}$ foi considerada lesão em órgão alvo pela 7ạ Diretriz Brasileira de Hipertensão Arterial, ${ }^{58}$ entretanto outras diretrizes admitem uma VOP maior que $10 \mathrm{~m} / \mathrm{s}$ como indicativo de lesão de órgão alvo, entre elas a Diretriz Europeia de 2013. ${ }^{25}$

Da mesma forma que a VOP, um Alx elevado, que é mais um parâmetro para avaliação de envelhecimento vascular, é preditor de risco de mortalidade por todas as causas e por causas cardiovasculares. Estudo identificou que um incremento de $10 \%$ no Alx aumenta o risco de mortalidade cardiovascular em $48 \%$ e mortalidade por todas as causas em $52 \% .{ }^{59}$

\section{PARÂMETROS CENTRAIS NA ESTRATÉGIA DE TRATAMENTO DA HIPERTENSÃO ARTERIAL}

Apesar da adequada redução da PA periférica com tratamento anti-hipertensivo, os resultados sobre desfechos clínicos mostraram diferença significante atribuída a efeitos pleiotrópicos dos anti-hipertensivos sobre as propriedades elásticas das grandes artérias, sobre a pressão aórtica central e a VOP ${ }^{60}$ A Tabela 1 mostra os efeitos de diferentes classes de anti-hipertensivos sobre a hemodinâmica central. ${ }^{61}$

\section{AS DIFERENTES CLASSES DE DROGAS E SEUS EFEITOS NOS PARÂMETROS CENTRAIS}

O estudo CAFE demonstrou o efeito da associação atenolol/hidroclorotiazida sobre a pressão central. Para o mesmo valor de PA periférica, os valores médios da PAc eram maiores quando comparados ao grupo anlodipina/perindopril. ${ }^{44} \mathrm{Di}$ ferentemente dos betabloqueadores de primeira e segunda geração, o nebivolol com efeito vasodilatador mediado pelo aumento do óxido nítrico, e o carvedilol com efeito alfa e beta bloqueador, promoveram uma maior redução da PAc e da VOP em comparação com o atenolol. ${ }^{62}$

Em modelos experimentais, os bloqueadores dos canais de cálcio (BCC) reduzem o estresse oxidativo e diminuem a PAc. ${ }^{63} \mathrm{O}$ estudo AORTA comparou a adição de azelnidipina ou anlodipina em hipertensos em uso de olmesartana e demonstrou que o grupo que recebeu azelnidipina obteve uma maior redução da PAc e do Aix. ${ }^{64,65}$

A redução da PAc demonstrada em estudos comparativos com inibidores da enzima conversora da angiotensina (IECA) pode ser atribuída a possíveis mecanismos envolvendo redução da complacência, diminuição do estresse oxidativo, recomposição estrutural da parede vascular, relação colágeno/ elastina, efeito anti-inflamatório e consequente relaxamento da musculatura lisa vascular. ${ }^{61,66,67} \mathrm{~A}$ valsartana e o captopril reduzem de forma semelhante a PAc e a VOP. ${ }^{68} \mathrm{O}$ estudo EXPLOR comparou valsartana/anlodipina versus anlodipina/ atenolol e para a mesma redução da PA periférica, a PAc e a VOP apresentaram maior redução no grupo da valsartana/ amlodipina. ${ }^{61,69}$ Estudos com outros bloqueadores dos receptores AT1 mostraram resultados semelhantes. ${ }^{61,70,71}$

Diuréticos parecem não ter efeito benéfico sobre a hemodinâmica central. ${ }^{72,73}$

O efeito dos nitratos sobre a redução da PAc é atribuído ao relaxamento da musculatura lisa das artérias de médio calibre. $\mathrm{O}$ mononitrato de isossorbida também foi avaliado em hipertensos e demonstrou maior redução da PAc do que da PA periférica, e maior redução do Alx sem modificação significante da frequência cardíaca. ${ }^{61}$ Por outro lado, os nitratos não influenciam a VOP, dessa forma, o efeito sobre a rigidez arterial é discreto. ${ }^{74}$

Revisão sistemática de 9 estudos elegíveis sobre efeito das

Tabela 1. Efeitos das diferentes classes de anti-hipertensivos na hemodinâmica central.

\begin{tabular}{|c|c|c|c|c|c|c|}
\hline Classes de anti-hipertensivos & PCaS & PCaD & Amplificação & Reflexão & VOPcf & PAP \\
\hline Betabloqueadores & $\uparrow \uparrow$ & $\leftrightarrow$ & $\downarrow$ & $\uparrow$ & $\leftrightarrow$ & $\downarrow$ \\
\hline Bloqueadores dos canais de cálcio & $\downarrow$ & $\downarrow / \hookleftarrow \rightarrow$ & $\uparrow$ & $\downarrow$ & $\downarrow$ & $\downarrow$ \\
\hline $\begin{array}{l}\text { Inibidores da enzima conversora } \\
\text { da angiotensina }\end{array}$ & $\downarrow \downarrow$ & $\downarrow$ & $\uparrow$ & $\downarrow$ & $\downarrow$ & $\downarrow$ \\
\hline $\begin{array}{l}\text { Bloqueadores dos receptores AT1 } \\
\text { da angiotensina II }\end{array}$ & $\downarrow$ & $\downarrow / \leftarrow \rightarrow$ & $\uparrow / \leftarrow \rightarrow$ & $\downarrow$ & $\downarrow$ & $\downarrow$ \\
\hline Diuréticos & $\leftrightarrow$ & $\leftrightarrow$ & $\leftrightarrow \rightarrow / \downarrow$ & $\leftrightarrow$ & $\leftrightarrow$ & $\downarrow$ \\
\hline Nitratos & $\downarrow$ & $\downarrow$ & $\downarrow$ & $\downarrow$ & $\leftrightarrow$ & $\leftrightarrow / \downarrow$ \\
\hline
\end{tabular}

PCaS: pressão central aórtica sistólica; PCaD: pressão central aórtica diastólica; VOPcf: velocidade da onda de pulso carotídeo-femoral; PAP: pressão arterial periférica. 
estatinas na rigidez arterial, cuja indicação mais frequente para a terapia com estatina foi hiperlipidemia com ou sem outros fatores de risco cardiovasculares, mostrou que houve redução consistente da VOP na maioria dos estudos. ${ }^{75}$

Artigo de revisão cita algumas drogas que definitivamente melhoram a rigidez arterial: IEAC, BRA, BCC, antagonista da aldosterona, espironolactona, glitazonas (pioglitazona) e aminoguanidina (inibidor de AGE's). Apontam outros fármacos que melhoram rigidez arterial na maioria dos estudos: betabloqueadores de terceira geração, estatinas, metformina, cloreto de alagebrium (inibidor de AGE's), sitaxsentan (antagonista do receptor da endotelina A) e corticosteroides. Relatam drogas com efeitos conflitantes: nitratos, eplerenona, rosiglitazona, anti-TNFa e AAS e drogas com efeito neutro: diuréticos e omapratilato. ${ }^{76}$ Estudos recentes mostram que os inibidores de SGLT2, empagliflozina, dapagliflozina e canagliflozina, são capazes de reduzir a PA periférica, mas sobretudo melhoram a função endotelial e rigidez arterial cujos efeitos são independentes da redução da PA, sugerindo um benefício direto na vasculatura, possivelmente mediado pela redução do estresse oxidativo. ${ }^{77-80}$

\section{REFERÊNCIAS}

1. Townsend RR, Wilkinson IB, Schiffrin EL et al. Recommendations for improving and standardizing vascular research on arterial stiffness: A scientific statement from the American Heart Association. Hypertension. 2015; 66(3):698-722.

2. Laurent S, Cockcroft J, Van Bortel L, et al. Expert consensus document on arterial stiffness: methodological issues and clinical applications. Eur Heart J. 2006; 27(21):2588-605.

3. Fan X, Zhu M, Chi C, et al. Association of arteriosclerosis and/ or atherosclerosis with hypertensive target organ damage in the communitydwelling elderly Chinese: the Northern Shanghai Study. Clin Interv Aging. 2017; 12:929-36.

4. Palatini P, Casiglia E, Gąsowski J, et al. Arterial stiffness, central hemodynamics, and cardiovascular risk in hypertension. Vasc Health Risk Manag. 2011; 7:725-39.

5. Protogerou AD, Safar ME, Papaioannou TG, et al. The combined effect of aortic stiffness and pressure wave reflections on mortality in the very old with cardiovascular disease: the PROTEGER Study. Hypertens Res. 2011;34:803-8.

6. Sougawa Y, Miyai N, Utsumi M, Miyashita K, Takeda S, Arita M. Brachialankle pulse wave velocity in healthy Japanese adolescents: reference values for the assessment of arterial stiffness and cardiovascular risk profiles. Hypertens Res. 2020;43:331-41.

7. Sun P, Yang Y, Cheng G, et al. Noninvasive central systolic blood pressure, not peripheral systolic blood pressure, independently predicts the progression of carotid intimamedia thickness in a Chinese community-based population. Hypertens Res. 2019;42:392-9.

8. Vlachopoulos C, Aznaouridis K, O'Rourke MF, Safar ME, Baou K, Stefanadis C. Prediction of cardiovascular events and all-cause mortality with arterial stiffness: a systematic review and meta- analysis. Eur Heart J. 2010;31:1865-71.

9. McEniery CM, Cockcroft JR, Roman MJ, Franklin SS, Wilkinson IB. Central blood pressure: current evidence and clinical importance. Eur Heart J. 2014;35:1719-25.

10. Ben-Shlomo Y, Spears M, Boustred C, et al. Aortic pulse wave velocity improves cardiovascular event prediction: an individual participant metaanalysis of prospective observational data from 17,635 subjects. J Am Coll Cardiol. 2014;63:636-46.

11. Mozos I, Malainer C, Horbańczuk J, et al. Inflammatory Markers for Arterial Stiffness in Cardiovascular Diseases. Front Immunol. 2017; 8:1058.

12. Lusis AJ. Atherosclerosis. Nature. 2000;407(6801):233-41.

13. Maksuti E, Westerhof N, Westerhof BE, Broome M, Stergiopulos N. Contribution of the Arterial System and the Heart to Blood Pressure during Normal Aging - A Simulation Study. PLoS One. 2016;11(6):e0157493.

14. Avolio A. Arterial Stiffness. Pulse. 2013; 1(1):14-28. http://dx.doi.

15. Brozovich FV, Nicholson CJ, Degen CV, Gao YZ, Aggarwal M, Morgan KG. Mechanisms of Vascular Smooth Muscle Contraction and the Basis for Pharmacologic Treatment of Smooth Muscle Disorders. Pharmacol Rev. 2016; 68(2):476-532.

\section{CONCLUSÃO}

As evidências apresentadas na literatura mostram a PA braquial como um forte preditor de eventos cardiovasculares e que o emprego de medicamentos anti-hipertensivos para a sua redução está associado a um melhor prognóstico. Por outro lado, evidências mais recentes apontam uma maior representatividade da pressão aórtica central na ocorrência dos eventos diretamente relacionados ao coração, rins e cérebro.

As técnicas não invasivas de medição dos parâmetros centrais, como a PAc, VOP e Alx, permitem um diagnóstico confiável do aumento da rigidez arterial. Elas são de fácil aplicação na prática clínica e estudos clínicos especializados acumulam claras evidências que a avaliação destes parâmetros estão fortemente relacionados aos desfechos cardiovasculares.

A adoção dos métodos não invasivos de determinação dos parâmetros centrais na rotina de acompanhamento dos nossos pacientes, certamente permitirá o emprego de uma terapêutica com foco no antienrejecimento arterial.

16. Kroeker EJ, Wood EH. Comparison of simultaneously recorded central and peripheral arterial pressure pulses during rest, exercise and tilted position in man. Circ Res. 1955; 3:623-632.

17. Nichols WW O'Rourke MF. McDonald's Blood Flow in Arteries: Theoretic Experimental and Clinical Principles. 5th ed., London: Edward Arnold, 2005.

18. McEniery CM, Yasmin, Hall IR, Qasem A, Wilkinson IB, Cockcroft JR. Normal vascular aging: differential effects on wave reflection and aortic pulse wave velocity the anglo-cardiff collaborative trial (ACCT). J Am Coll Cardiol. 2005;46:1753-60.

19. Van Bortel LM, Balkestein EJ, van der Heijden-Spek JJ, et al. Non-invasive assessment of local arterial pulse pressure: comparison of applanation tonometry and echo-tracking. J Hypertens. 2001;19:1037-44.

20. Mekhail AM, Day LM, Goodhart AK, Wilkinson IB, McEniery CM. Noninvasive estimates of central systolic blood pressure: Comparison of the Centron cBP301 and SphygmoCor devices. Artery Res. 2012;6:109-13.

21. Climie RE, Schultz MG, Nikolic SB, Ahuja KD, Fell JW, Sharman JE. Validity and reliability of central blood pressure estimated by upper arm oscillometric cuff pressure. Am J Hypertens. 2012; 25:414-20.

22. Rezai MR, Goudot G, Winters C, Finn JD, Wu FC, CruickshankJK. Calibration mode influences central blood pressure differences between SphygmoCor and two newer devices, the Arteriograph and Omron HEM-9000. Hypertens Res. 2011;34:1046-51.

23. Guilcher A, Brett S, Munir S, Clapp B, Chowienczyk PJ. Estimating central SBP from the peripheral pulse: influence of waveform analysis and calibration error. J Hypertens. 2011;29:1357-66.

24. Papaioannou TG, Karageorgopoulou TD, Sergentanis TN, et al. Accuracy of Commercial Devices and Methods for Noninvasive Estimation of Aortic Systolic Blood Pressure a Systematic Review and Meta-Analysis of Invasive Validation Studies. J Hypertens. 2016;34(7):1237-48.

25. Mancia G, Fagard R, Narkiewicz K, et al. 2013 ESH/ESC Guidelines for the management of arterial hypertension: The Task Force for the management of arterial hypertension of the European Society of Hypertension (ESH) and of the European Society of Cardiology (ESC). Eur Heart J. 2013 Jul;34(28):2159-219.

26. Mitchell GF. Aortic stiffness, pressure and flow pulsatility, and target organ damage. J Appl Physiol (1985). 2018; 125(6):1871-80.

27. Kaess BM, Rong J, Larson MG, et al. Aortic stiffness, blood pressure progression, and incident hypertension. JAMA. 2012; 308(9):875-81.

28. Vlachopoulos C, Aznaouridis K, Stefanadis C. Prediction of cardiovascular events and all-cause mortality with arterial stiffness: a systematic review and meta-analysis. J AmColl Cardiol. 2010; 55(13):1318-27.

29. Ben-Shlomo Y, Spears M, Boustred C, et al. Aortic pulsewave velocity improves cardiovascular event prediction: an individual participant metaanalysis of prospective observational data from 17,635 subjects. J AmColl Cardiol. 2014;63(7):636-46.

30. Protogerou A, Blacher J, Stergiou GS, Achimastos A, Safar ME. Blood pressure response under chronic antihypertensive drug therapy: the role of aortic stiffness in the REASON (Preterax in Regression of Arterial Stiffness in 
a Controlled Double-Blind) study. J AmColl Cardiol. 2009;53(5):445-51.

31. Guerin AP, Blacher J, Pannier B, Marchais SJ, Safar ME, London GM. Impact of aortic stiffness attenuation on survival of patients in end-stage renal failure. Circulation. 2001;103(7):987-92.

32. Vlachopoulos C, Terentes-Printzios D, Laurent S, et al. Association of Estimated PulseWave Velocity With Survival. A Secondary Analysis of SPRINT. JAMA Network Open. 2019;2(10): 1912831.

33. de Luca N, Mallion JM, O'Rourke MF, et al. Regression of left ventricular mass in hypertensive patients treated with perindopril/indapamide as a firstline combination: the REASON echocardiography study. Am J Hypertens. 2004;17:660-7.

34. Bortolotto LA. Pressão central: como interpretar na prática clínica. Rev Bras Hipertens. 2009;16(1):46-7.

35. Pauca AL, Wallenhaupt ST, Kon ND, Tucker WY. Does radial artery pressure accurately reflect aortic pressure. Chest. 1992;102:168-75.

36. O'Rourke MF. The arterial pulse in health and disease. Am Heart J. 1991;82:687-702.

37. Chen $\mathrm{CH}$, Nevo E, Fetics B, Pak PH, Yin FC, Maughan WL, Kass DA.. Estimation of central aortic pressure waveform by mathematical transformation of radial tonometry pressure: validation of generalised transfer function. Circulation 1997; 95:1827-36.

38. O'Rourke MF, Gallagher DE. Pulse wave analysis. J Hypertens. 1996;14(Suppl 5):S147-S157

39. Nichols WW, Avolio AP, Kelly RP, O'Rourke MF. Effects of age and of hypertension on wave travel and reflections. In: O'Rourke MF, Safar ME, Dzau V (eds). Arterial Vasodilation: Mechanisms and Therapy. Arnold: London, 1993.

40. O'Rourke MF, Kelly RP. Wave reflection in the systemic circulation and its implication in ventricular function in man. J Hypertens. 1993;11:327-37.

41. Drzewiecki GM, Melbin J, Noordergraaf A. Arterial tonometry: review and analysis. J Biomech. 1983;16:141-53.

42. Kemp CRW, Seibenhofer A, Williams B. Occult myocardial ischaemia in young hypertensive subjects without clinical evidence of ischaemic heart disease. J Hum Hypertens. 1999;13:5-6.

43. Kelly RP. Pharmacological potential for reversing the ill effects of ageing and of arterial hypertension on central aortic systolic pressure. J Hypertens. 1992;10(Suppl 6):97-100.

44. Williams B, O'Rourke M. Differential impact of blood pressure-lowering drugs on central aortic pressure and clinical outcomes: principal results of the Conduit Artery Function Evaluation (CAFE) Study. Circulation. 2006;113:1213-25.

45. Townsend RR, Wilkinson IB, Schiffrin EL, etal: American Heart Association Council on Hypertension. Recommendations for improving and standardizing vascular research on arterial stiffness: a scientific statement from the American Heart Association. Hypertension. 2015;66(3):698-722.

46. van Sloten TT, Schram MT, van den Hurk K, et al. Local stiffness of the carotid and femoral artery is associated with incident cardiovascular events and allcause mortality: the Hoorn study. J Am Coll Cardiol. 2014; 63(17):1739-47.

47. Lacy PS, O'Brien DG, Stanley AG, Dewar MM, Swales PP, Williams B. Increased pulse wave velocity is not associated with elevated augmentation index in patients with diabetes. J Hypertens. 2004;22(10):1937-44.

48. Jerrard-Dunne P, Mahmud A, Feely J. Ambulatory arterial stiffness index, pulse wave velocity and augmentation index--interchangeable or mutually exclusive measures? J Hypertens. 2008;26(3):529-34.

49. Townsend RR, Wilkinson IB, Schiffrin EL, et al; American Heart Association Council on Hypertension. Recommendations for improving and standardizing vascular research on arterial stiffness: a scientific statement from the American Heart Association. Hypertension. 2015;66(3):698-722.

50. Safar ME, Levy BI, Struijker-Boudier H. Current perspectives on arterial stiffness and pulse pressure in hypertension and cardiovascular diseases. Circulation. 2003;107(22):2864-9.

51. Mikael LR, Paiva AMG, Gomes MM, Sousa ALL, Jardim PCBV, Vitorino PVO, Euzébio MB, Sousa WM, Barroso WKS. Envelhecimento vascular e rigidez arterial. Arq Bras Cardiol. 2017;109(3):253-8.

52. Vlachopoulos $C$, Xaplanteris $P, A b o y a n s ~ V$, et al. The role of vascular biomarkers for primary and secondary prevention. A position paper from the European Society of Cardiology Working Group on peripheral circulation: Endorsed by the Association for Research into Arterial Structure and Physiology (ARTERY) Society. Atherosclerosis. 2015;241(2):507-32.

53. Mancia G, Fagard R, Narkiewicz K, et al. 2013 ESH/ESC Guidelines for the management of arterial hypertension: the Task Force for the management of arterial hypertension of the European Society of Hypertension (ESH) and of the European Society of Cardiology (ESC). J Hypertens. 2013; 31(7):1281-357.

54. Malachias MV, Souza WK, Plavnik FL, et al; Sociedade Brasileira de Cardiologia. 7 a Diretriz brasileira de hipertensão arterial. Arq Bras Cardiol. 2016; 107(3 supl 3):1-83.

55. Henskens LHG, Kroon AA, Oostenbrugge Van, et al. Increased aortic pulse wave velocity is associated with silent cerebral small-vessel disease in hypertensive patients. Hypertension. 2008; 52:1120-6.

56. Guerin AP, J Blacher, B Pannier, SJ Marchais, Safar ME, London GM. Impact of Aortic Stiffness Attenuation on Survival of Patients in End-Stage Renal Failure; Circulation. 2001:103;987-92.

57. Hausvater A, Giannone T, Sandoval YH, et al. The association between preeclampsia and arterial stiffness. J Hypertens. 2012;30(1):17-33.

58. Malachias, MVB, Franco, RJS, Forjaz, CLM, et al. 7a Diretriz Brasileira de Hipertensão Arterial. Arq Bras Cardiol 2016; 107(3Supl.3):1-83.

59. London GM, Blacher J, Pannier B, Guérin AP. Arterial wave reflections and survival in end-stage renal failure. Hypertension. 2001;38:434-8.

60. Lewington S, Clarke R, Qizilbash N, Peto R, Collins R. Age-specific relevance of usual blood pressure to vascular mortality: a meta-analysis of individual data for one million adults in 61 prospective studies. Lancet. 2002;360(9349):1903-13.

61. Brandão AA, Amodeo C, Alcântara C, et al. I Posicionamento Luso-Brasileiro de Pressão Arterial Central. Arq Bras Cardiol. 2017;108(2):100-8.

62. Vaz-de-Melo RO, Giollo-Junior LT, Martinelli DD, et al. Nebivolol reduces central blood pressure in stage I hypertensive patients: experimental single cohort study. Sao Paulo Med J. 2014;132(5):290-6.

63. Williams B, Lacy PS, Thom SM, et al. Differential impact of blood pressurelowering drugs on central aortic pressure and clinical outcomes: principal results of the Conduit Artery Function Evaluation (CAFE) study. Circulation. 2006;113(9):1213-25.

64. Takami T, Saito Y. Effects of Azelnidipine plus OlmesaRTAn versus amlodipine plus olmesartan on central blood pressure and left ventricular mass index: the AORTA study. Vasc Health Risk Manag. 2011;7:383-90.

65. Takami T, Saito Y. Azelnidipine plus olmesartan versus amlodipine plus olmesartan on arterial stiffness and cardiac function in hypertensive patients: a randomized trial. Drug Des Devel Ther. 2013; 7:175-83.

66. Protogerou AD, Stergiou GS, Vlachopoulos C, Blacher J, Achimastos A. The effect of antihypertensive drugs on central blood pressure beyond peripheral blood pressure. Part II: Evidence for specific class-effects of antihypertensive drugs on pressure amplification. Curr Pharm Des. 2009;15(3):272-89.

67. Manisty $\mathrm{CH}$, Hughes AD. Meta-analysis of the comparative effects of different classes of antihypertensive agents on brachial and central systolic blood pressure, and augmentation index. Br J Clin Pharmacol. 2013;75(1):79-92.

68. London GM, Pannier B, Vicaut E, et al. Antihypertensive effects and arterial haemodynamic alterations during angiotensin converting enzyme inhibition. J Hypertens. 1996;14(9):1139-46.

69. Boutouyrie P, Achouba A, Trunet P, Laurent S. Amlodipine-valsartan combination decreases central systolic blood pressure more effectively than the amlodipine-atenolol combination: the EXPLOR Study. Hypertension. 2010; 55(6):1314-22.

70. Agnoletti D, Zhang Y, Borghi C, Blacher J, Safar ME. Effects of antihypertensive drugs on central blood pressure in humans: a preliminary observation. Am J Hypertens. 2013; 26(8):1045-52.

71. Asmar R. Effect of telmisartan on arterial distensibility and central blood pressure in patients with mild to moderate hypertension and Type 2 diabetes mellitus. J Renin Angiotensin Aldosterone Syst. 2001; 2 Suppl 2:S8-11.

72. Morgan T, Lauri J, Bertram D, Anderson A. Effect of different antihypertensive drug classes on central aortic pressure. Am J Hypertens. 2004;17(2):118-23.

73. Mackenzie IS, McEniery CM, Dhakam Z, Brown MJ, CockcroftJR, Wilkinson IB. Comparison of the effects of antihypertensive agents on central blood pressure and arterial stiffness in isolated systolic hypertension. Hypertension. 2009:54(2):409-13.

74. Stokes GS, Barin ES, Gilfillan KL. Effects of isosorbide mononitrate and AII inhibition on pulse wave reflection in hypertension. Hypertension. 2003; 41(2):297-301.

75. Rizos EC, Agouridis AP, Elisaf MS. The effect of statin therapy on arterial stiffness by measuring pulse wave velocity: a systematic review. Curr Vasc Pharmacol. 2010; 8(5):638-44

76. Janić M, Lunder M, Šabovič M. Arterial stiffness and cardiovascular therapy. Biomed Res Int. 2014;2014:621437.

77. Maliha G, Townsend RR. SGLT2 inhibitors: their potential reduction in blood pressure. J Am Soc Hypertens. 2015;9(1):48-53. .

78. Cabrera-Sole R, Lucas CT, Rivera LU, Ruiz SG, Aguilera M. Hypertensive, Type II Diabetic and Obese Patients, Are Better Controlled and Reduce the Number of Drugs When Empagliflozin is Added to Standard Treatment Improving Also Arterial Stiffness and Central Blood Pressures. Hypertension. 2017;70:AP457.

79. Solini A, Giannini L, Seghieri M et al. Dapagliflozin acutely improves endothelial dysfunction, reduces aortic stiffness and renal resistive index in type 2 diabetic patients: a pilot study. Cardiovasc Diabetol, 2017; 16:138.

80. Ramirez AJ, Sanchez MJ, Sanchez RA. blood pressure and arterial stiffness effects of canagliflozin or perindopril. J Hypertens. 2019;37(3):636-642. 